\title{
Wireless Capsule Video Endoscopy: A New Diagnostic Method for Aortoduodenal Fissure
}

B. González-Suárez ${ }^{1}$, C. Guarner ${ }^{1}$, J. R. Escudero ${ }^{2}$, E. Viver ${ }^{2}$, J. Palmer ${ }^{3}$, J. Balanzó ${ }^{1}$

${ }^{1}$ Department of Gastroenterology, Hospital de Sant Pau, Barcelona, Spain ${ }^{2}$ Department of Angiology, Hospital de Sant Pau, Barcelona, Spain ${ }^{3}$ Department of Radiology,

Hospital de Sant Pau, Barcelona, Spain

\section{Corresponding Author}

B. González-Suárez, M.D.

Department of Gastroenterology

Hospital de Sant Pau

Sant Antoni M $\mathrm{M}^{\mathrm{a}}$ claret 167

08025 Barcelona

Spain

Phone: + 34-93-291-9139

Fax: + 34-93-291-9278

E-mail: mgonzalezs@hsp.santpau.es

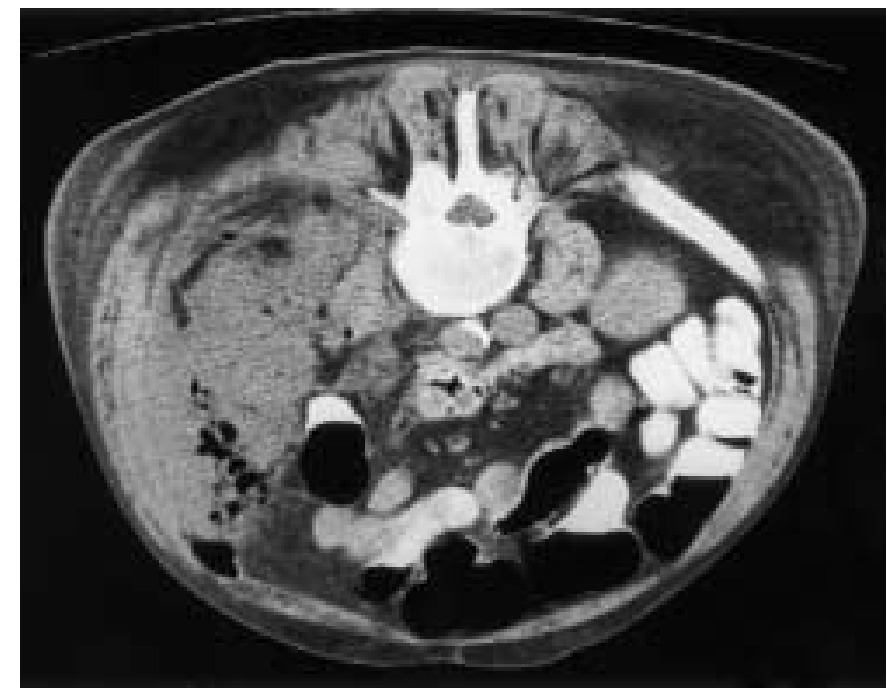

Figure 1 A 66-year-old man with a history of aortobifemoral bypass 5 years previously was admitted to our hospital with abdominal pain and fever. Abdominal computed tomography (CT) was carried out, revealing the presence of gas involving the aortic prosthesis, and a large retroperitoneal abscess. Antibiotic therapy was initiated, and the abscess was drained under radiographic control. The patient was scheduled for surgical intervention $48 \mathrm{~h}$ later, with a suspicion of aortic-enteric fissuration.

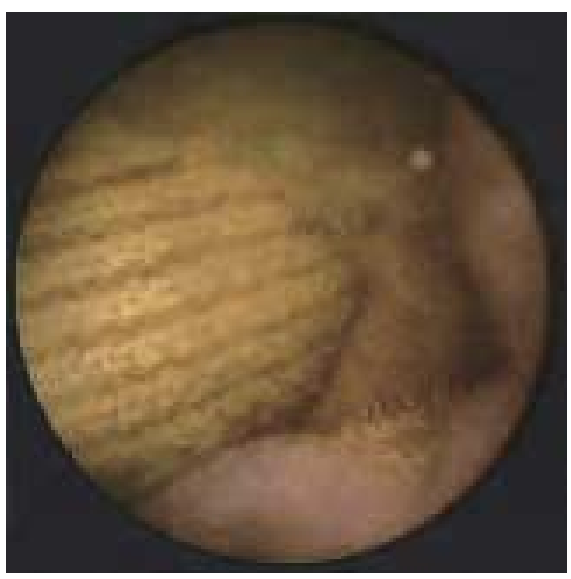

Figure 2 Meanwhile, wireless capsule video endoscopy was carried out to confirm the diagnosis in this patient, owing to the high risk of the intervention. Surprisingly, the wall of the small intestine in the ascending duodenum had been replaced by the Dacron prosthesis, producing the imaging appearance shown here. This unexpected finding was confirmed during open abdominal surgery. 